\title{
Fashioning Embodied Interfaces: Open Wearables Crafting
}

\author{
Valérie Lamontagne \\ Concordia University, Design \& Computation Arts, 1515 St. Catherine St. West, Montreal, \\ Quebec, Canada H3G 2W1 \\ valerie@3lectromode.com
}

\begin{abstract}
This paper investigates the role of the designer in the "opening" of culture in fashion and technology. In particular it explores the convergence of "open practices" in vanguard technologies and fabrication processes found in the history of Modernist fashion, as well as recent popular uses of technology, and engineering, and more specifically wearables design practices.
\end{abstract}

Keywords: Wearables, fashion, open design, embodiment, interface culture, textiles, prototyping, performance.

\section{Introduction}

Two narratives that contextualize the relationship between open culture, technology, and the history of fashion are proposed in this paper.

The first narrative is rooted in turn-of-the-century Paris, where the concept and role of the fashion designer was birthed in tandem with unheralded innovations in the manufacturing industry. This transformation changed and challenged our relationship with garments, the changes stemming from shifts in clothing's cultural capital and the processes associated with their production.

The second narrative stream explores the expanding landscape of current hybrid techno-artistic practices of wearables design and production-a field combining technical know-how from various fields, including engineering, textile innovation, fashion production and sartorial expression. The common thread tying these stories together is found in the increased access to materials, technologies and skill-practices since the modern era. Access to materials, tools, and information figure prominently in the drama of how fashion and technology came to be "opened up" through open design practices.

\section{Fashion and Engineering}

Fashion and engineering, as practiced-based disciplines, have more in common than is initially visible. To begin with, both are practices rooted in research and iteration that participate in a continuum of evolution and constant transformation. The products of fashion and technology are transient, trend-driven, technology-based and irrevocably "of the moment." 
Fashion and technology are also both children of the modern era. Technology and fashion as we know it emerged at the turn of the century as a result of rapid change in material and industrial innovations, social and economic events, and mass-market transportation networks $[1 ; 2]$.

As cultural products, fashion and technology define and materially embody the times during which they are designed and used. As cultural artifacts, they are beacons of our desires, projected fantasies, hopes and beliefs. Fashion and technology crystallize the contemporary in an ever-unfolding and insatiable process of production. I will also argue that, perhaps due to their fleeting and evanescent nature, the survival and constant reinvention of fashion and technology is deeply entwined with open culture practices in which the sharing of information, techniques and processes are key.

\subsection{Modernism, Sewing and Fashion: Poiret}

Before 1900, there were no real fashion designers. There were garment makers or seamstresses who gained a reputation by executing the sartorial visions of their clients, making to-order garments based on general stylistic trends or rank $[1 ; 2]$. However, they did not consider themselves artists or creative individuals. All this changed in Paris at the early turn of the century, when couturiers such as Paul Poiret marketed and crafted identities as "artists," as opposed to mere "makers." It was Poiret who, in 1904, pronounced himself a fashion "designer," claiming the position of style arbitrator [3; 4; 5]. Having worked at the House of Worth (1990-1004), Poiret was the first to align his craft with artistic practices such as Modern painting and sculpture that were coming to the fore in Paris and Europe at the time. In this climate of economic affluence, rapid social change and artistic dynamism, Poiret cast himself as a fashion innovator, gaining international influence and markets across Europe and America [3]. Within his active career (1903-1929), Poiret was dubbed "The King of Fashion" and "Le Magnifique." He was prolifically active in fashion, perfume, film and theatre costumes, and fashion training schools, as well as the international trunk shows that brought him to America numerous times. At peak of his influence, Poiret's styles and opinions made numerous news headlines and transformed the ways in which his clients and society at large viewed fashion's role in society [4].

\subsection{Paris and Fashion}

Not coincidentally, the upgrading of the garment "maker" to that of a "designer" and "artist" arrived at a time of great technological and material transformation that affected the social and tangible make-up of everyday life [6; 7]. At the turn of the century, numerous technological changes transformed the social sphere, including the proliferation of transportation channels-from trains and automobiles to steampowered ships - that facilitated the exchange of ideas, styles and social groups across a larger geographic area. Also in this time period, city architecture was completely transformed. Baron Georges-Eugène Haussmann re-tooled the Parisian cityscape, adding wide boulevards, street lights and clean, safe paved streets and alleys; these developments changed the way that the city was used and by whom. The modern 
city's infrastructure of boulevards, civic parks and interior shopping arcades encouraged greater urban mobility, especially among the women who could now walk though the city safely, without social stigma.

These changes in transportation and urban design-along with an increase in economic prosperity and leisure time on the part of a growing middle classcontributed to the increased importance of personal sartorial expression, as well as the exploration of fashion innovation and variety $[1 ; 8]$. The modern era built a need for individuals to be seen as being personally expressive, combined with a desire to display newly acquired wealth, social standing and stylistic "savoir faire." During this same modernist era were cemented the legacies of today's major Parisian couture houses. Many now-ubiquitous couture labels had humble beginnings as "makers." These artisans and craft-focused ateliers later evolved into significant style arbitrators and international economic powerhouses. Louis Vuitton, for example, was known as a luggage maker who dabbled in doll clothes up until the mid-twentieth century. Coco Chanel, the revolutionary designer who introduced "poor" materials and sportswear cuts to fashion, worked primarily as a seamstress until the First World War. However, is was Poiret who daringly embarked on a journey of making fashion fashionable for its own sake. Influenced by the bohemian scene of artists living in Paris at that time, Poiret is known for having done away with corsets and embraced Oriental themes and textiles; he was also influential in introducing the public to works of contemporary artists such as Raoul Dufy, featured in the couturier's textile designs, party invitations and set designs for fashion shows. In fact, Poiret is one of the inventors of today's runway performance. Heavily inspired by theatre work, he mounted theatrical showcases of his fashion designs on custom stages in his couture house as well as department stores such as Gimbels in New York City [3].

\subsection{Trademarks and Logos}

By all accounts, Poiret was very media savvy for his era. He took every opportunity to promote his name and brand. When he discovered that his designs were being forged in America and at home, he became president of La Chambre Syndicale de la Couture, laying the groundwork to protect intellectual property design in fashion. Many other growing couture houses were also becoming more and more invested in protecting their brand. This legalistic push to protect the integrity of creative elements, until then unseen in the garment industry, led to the creation of logo copyrights as seen today. To this day it is logos and trademarks that are prominently protected, much more than the aesthetic cut or style of a garment or accessory, though there have been recent cases to the contrary, such as Christian Louboutin trying to protect his famous red under heel.

\subsection{Pattern-Making and Distribution}

The rise of the "designer" occurs, interestingly, in parallel with both the proliferation of home sewing machines and an increased access to products such as textiles 


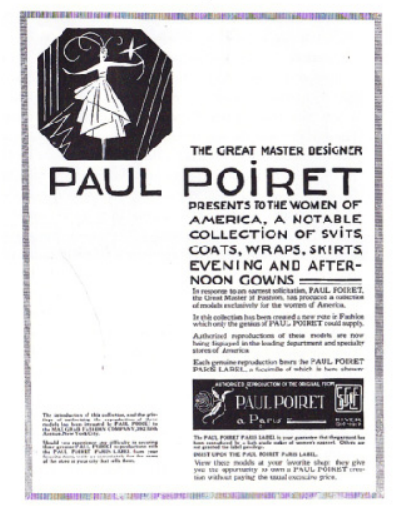

Fig. 1. Paul Poiret advertisement 1912

imported from various parts of the world, giving everyday "makers" an opportunity to craft their own design [9]. This meant an increase in makers, as well as access to the tools to make things at a higher level of quality and customization. Therefore, it wasn't only the logos by Poiret (and other designers) that were being copied. In fact, professional seamstresses and store manufacturers were reproducing entire styles and patterns-sometimes as legal and "official" copies. For Poiret and his contemporaries, a more radical approach had to be taken to protect the "intellectual property" of their designs and their status as "designers." The pirating of patterns occurred especially in America where Parisian styles were all the rage, and Poiret was considered the City of Light's reigning monarch. In an effort to stave off imitators, Poiret created one of the first official designer "patterns," sold and "authenticated" as an original "Paul Poiret" design. Although it was intended to protect the integrity of the designer, what Poiret had in fact inaugurated was the democratization of fashion as seen in designer patterns today from Vogue to McCalls. Instead of authenticating his products through their origins (made in his Paris atelier) or their logo, these patterns placed value on the design of the product, rather than the product itself. The design as opposed to the origin of making was most valued. Poiret can also be credited, via his proliferation of patterns, as the first populist DIY promoter of fashion, though he may not have thought of it this way.

\section{Open Design and Wearables}

The exciting ramifications of such a shift in thinking about fashion creativity, from a finished object to one which may be executed and potentially customized by others, inspired the values also at the heart of the avant-garde open design culture blossoming today. Design kits and DIY templates executed by individuals factor in and inevitably celebrate the vagrancies of different styles of interpretation, material choices, toolexploration and end results. Fashion patterns, along with numerous kits from multiple industries, became popularized at the start of the 20th century. One could order kits, instructions and materials to build such things from homes to radios, from socks to 
furniture. These original templates form the core of open culture thinking today, a revival of pre-post industrialism and craft engagement as described by Sennet [10]. According to Andersen and Gershenfeld, we are presently facing a new kind of postindustrial revolution of "making," enabling individuals to enter the chain of production on a small and personal scale [11;12].

Wearables, the result of the admixture of fashion and electronics, are closely aligned with the growing movement of open design practices and access to technology. Wearables has greatly benefited from a belief that the field has the potential to amalgamate contributions from many individuals and practices coming from the fields of engineering, electro-mechanical industries, textiles and fashion field. As well, the field of wearables and fashion-tech would not be where it is today without immense contributions by people working in electronics, craft, hobbyist and other forms of admixtures of tech-design experimentation that are increasingly opensourced and available via networks such as the Web. In this section I want to highlight how current innovation in wearables is a natural evolution of open design practices stemming from innovation and re-thinking in modern fashion, art and technological popularization.

\subsection{Materials}

The field of wearables would not be where it is today without the belief that artists and designers had something to contribute to technology. Coined in 1991 by Steve Mann at MIT, "wearables" as fashion tech has principally been a door through which the material experimentation of electronics could be elaborated in design contexts, often related to the body, as this is the site of technological exploration for wearables. Books such as "Physical Computing" by Tom Igoe from New York University's Interactive Telecommunications Program revolutionized the language of electronics, making it accessible to a whole new set of actors with art backgrounds and hacker mentalities [13]. In Canada, robotics pioneer Norm White at OCAD schooled computational innovators such as David Rokeby, forever changing the landscape of media arts practices from users of tools (such as video cameras) to makers of tools (such as circuits and programming languages).

Closer to the field of wearables, Leah Buechley developed the LilyPad Arduino platform, the first instance of adapting electronics for wearables. From an engineering perspective, Arduino is like a cake mix for arts electronics, bringing all essential ingredients together and simplifying the language. Since then, other companies such as Adafruit's Flora, Aniomagic and SparkFun have expanded the repertoire and accessibility of materials and technologies offered to users, making it even easier to customize electronics effects. Furthermore, "prêt-à-faire" (ready to make) DIY practices in fashion-incorporating the new production technologies of digital textile printers, 3D printing, and laser cut patterns-are being seen all over the runways, heralding a new way of conceiving of how to dress the body. In less than 10 years, we have seen the material landscape of wearable technologies not only expand but become dynamically accessible, affordable, and full of potential for creative "designerly" (as opposed to thinking that only engineering matters) results. 


\section{2 $\quad$ Access $=$ Knowledge + Tools}

Open design practices flourish with access to knowledge and tools-this means placing not only materials and tools within easy reach, but the practices, methods and knowledge that give ready hands access to creative solutions. There are two prominent areas of access: the first is through publishing, formerly the Diderot's Encyclopédie, now encountered in the everyday as how-to manuals, guide-books, and increasingly the Internet with its wealth of photo and video tutorials. Books such as Sabine Seymour's "Fashionable Technology" and "Functional Aesthetics," Syuzi Pakchyan's "FashioningTech," the collaborative "Open Softwear," and Otto von Busch's hacking couture guidebook "Becoming Fashion-able" have proven important in bringing wearables to an audience of novices. Furthermore, sites such as Instructables and Craft, FashioningTech, and Etsy feature a wide range of technology, craft-based tutorials and ideas for materials, methods, providing inspiration and a community to share it with.

Other websites such as Thingiverse share files for the emerging practices of 3D printing and other forms of machine-tooled and 3-dimensional object making. Tangible meeting and working sites and fabrication laboratories (or FabLabs), such as ProtoSpace (Utrecht, Netherlands) and Open Design City (Berlin) as well as labs such as V2_ (Rotterdam) have made a significant change in the availability of access to machines such as 3D printers and laser cutters, as well as bringing individuals into contact with a community of technical and computational experts. Of course, festivals, fairs and events such as MakerFaire, SIGGRAPH, SXSW, Transmediale, FutureEverything and ISEA provide great opportunities to share knowledge and skills and meet the actors involved in the global shift of sharing design expertise. In short, the design, art and technical world is producing an increasing number of nodes of information, sharing, encounters, testing, advice and hands-on material making.

\subsection{Social Adaptation $=$ Made $4 \mathrm{U}$}

Another area of interest is how remote and online platforms are proposing ways for designers and consumers to collaborate in creating open designs. Using as a template the pattern adjustments and choices of textiles or embellishments that sewing patterns provide, online and rapid prototyping technologies offer new opportunities for social adaptations. Customization and user-input platforms invite experts and novices alike to reproduce, modify, improve, customize, and be inspired by the work of others. This type of network and platform fosters co-creation, and "personal design nodes" where the shape and making of design can be seamlessly personalized and adapted to use or aesthetic preference, It is both about the personal and the collective in as much as it solicits input from individuals for their needs and desires while also keeping the practice and knowledge open-ended enough for collective contributions and specializations over time.

Products such as the user-generated, nature-inspired jewelry by Nervous System and Shapeways propose new and exciting design collaborations where the results unfold unexpectedly. From within fashion, companies such as Unitestyles propose 
platforms to customize their designs, while the über-rarefied Maison Martin Margiela has been inviting users since 2004 to adapt unfinished designs to their liking and post them online. Finally, computational couture mavens such as May Huang propose 3D algorithmic designs, which are also user-generated. These online platforms offer a way in which the consumer may become part of the design process - an invitation which can at times be daunting, yet exciting. Even in the event of a design failure, the consumer can better appreciate the importance of design and the power of networked and rapid prototyping technologies in making ideas tangible. These kinds of open access platforms have been thoroughly explored and documented at Amsterdam's Open Design Lab of the Waag Society where designers are encouraged to create "open” design for commerce [14].

\subsection{Unzipping Wearable Fashion}

Open design materials, knowledge and tools, as previously mentioned, have democratized and 'unzipped' wearables practice. Increasingly fashion-tech is making use of an increasing complex array of engineering and computational skills, sartorial knowhow and material experimentation, making the design studio more akin to a laboratory producing new aesthetics and technologies to transform the body. What were previously craft or technical-only niche groups are becoming increasingly mainstream-yet independent-hybrid tech-fashion design studios. The factory is no longer over "there" but rather down the street, or in our living rooms. Access to hightech tools and experts is 'industrializing' the practice of small scale designers, giving them more options to professionalize their craft through access to custom circuits, 3D printing, laser cutting, etc. These technical networks, both local and networked, help shape the hybridization of the wearables field by giving designers access to specialized knowledge and tools, resulting in the expansion of their material repertoire and craft expressiveness.

Increasingly, fashion is playing an important role in communicating who we arefrom the personal to the global — as it did at the turn of the $20^{\text {th }}$ century [15]. Fashion designers are regularly pushing the material envelope of what our sartorial choices can say about us. Contemporary designers such as Dutch Pauline van Dongen have collaborated with 3D printing companies like Freedom of Creation to create 3D printed shoes, while Iris van Herpen, also Dutch, has created entire garments out of 3D printing technologies. Anouk Wipprecht has collaborated with wearable art labs such as V2_to develop interactive garments that paint themselves, become transparent or are made of a cloud of smoke. These garment designs, though speculative for the moment, are forging a new material vision of what our garments can be and how they might convey who we are in a dynamic technological fashion world.

Meanwhile, other aspects of wearables are forging emerging tangible interfaces for technologies to be embedded in garments in a very concrete way. Diffus, a Danish design studio, has paired with Swiss lace company Forster-Rhoner to develop working prototypes in wearables that piggyback on century old know-how in lace making. Together they have fabricated solar-powered embroidered handbags that combine embellishment with functionality. Moon Berlin, a Berlin fashion label 


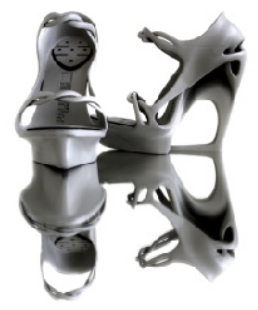

Fig. 2. Pauline van Dongen, Morphogenesis Shoe, 2011

exploiting light in their designs, have collaborated with the Fraunhofer IZM, an internationally reputable institution for the testing of technologies, to incorporate state-of-the-art stretchable circuits into their bespoke designs. All of these wearable designers are tapping into expertise and tools that are distributed on an increasingly collaborative scale. This is in part due to the many technical (garment, design, textile, electronics) types of expertise needed to create aesthetically and technically successful wearables. These are just a few of the examples of design and tech industries coming together to explore the potential of wearables. Often the collaborations are open exchanges, birthed out of necessity, stemming from this increasingly high-tech, hybrid, networked cottage/professional industry in which fashion innovation and electronics developments converge in professional yet highly craft-focused fashion-technology collaborations. Though the overlaps in knowledge fields of wearables at times come from divergent technical/artistic fields and economies, there is a desire for "sharing becoming a default standard," as noted in the Creative Common's recent anthology of interviews The Power of Open. This is a revolutionary moment for wearables and 3D objects - similar to the paradigm shift that occurred in the 2D world of desktop publishing in 1985-which we should embrace, share, contribute to and protect via Open Design philosophies and practices.

\section{3lectromode}

\subsection{DIY Kits}

I want to take this opportunity to speak about my own involvement in open design, via the 3lectromode platform. 3lectromode has a vision to innovate in the field of wearables by combining technology with customizable prêt-a-porter fashion. As a small group of practitioners working in the field of fashion and technology, we aim to inspire a future where wearables are democratized and aestheticized. We are interested in developing accessible wearables combining DIY technology with current fashion research and aesthetics. We are fascinated with the potential for technology to create new modalities of interaction between the body and its environment, and are interested in the expressive potential of technology to transform the experience derived from garment use from the poetic to the practical. 


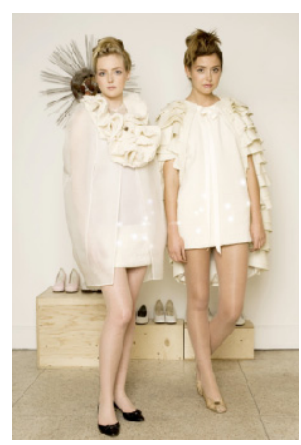

Fig. 3. 3lectromode, "Future Matter" 2012

\subsection{Prêt-à-Porter Tech}

Key to 3lectromode's design ethos is the desire to create a library of open sourced fashion designs which can be easily assembled as kits by anyone with an interest in wearables, electronics or fashion. The kits come complete with the printed garment, the necessary electronics and instructions, taking the guesswork out of electronics assembly while allowing the user to create a customized and fashionable design. Designs are printed on textile printers on which also include the layout of electronic schematics and sewing directions. The methods for assembling the electronic components of the wearable are integrated into the design and can be visually followed, much like a paint-by-numbers picture, without having to refer to a manual. Each piece is uniquely designed, and comes with customizable options for different print patterns, colours, models and sizes, giving the user-end designer the agency to creating his or her own iteration. Computational variations are also included to modify the LilyPad Arduino program. So far, 3lectromode designs have focused on integration of LEDs with various sensors, using the LilyPad Arduino platform for electronic components and programming. 3lectromode's kits are the perfect entry point into wearable technology because of their graphic visualization of electronics assembly methods, while also creating uniquely stylish and fashionable garments. In the process of testing out this open design platform, we at 3lectromode have been interested in integrating feedback from the user-end designers and welcoming collaborations on the sharing of techniques, designs and applications. Ultimately, while maintaining a stylistic curatorial vision true to 3lectromode, we are interested in seeing how people might hack and interpret our work in an open design fashion.

3lectromode as a platform was created for selfish reasons - to create wearables that one could wear in the everyday that have a higher design value component than some of the one-off (and admittedly fashion-starved) productions made in a crafting context. It's really the meeting of the sewing pattern and DIY circuit-design used to create recipes for making fashion that is at the heart of 3lectromode. Our designs are somewhere between a prototype for wearables and a way of having engineers discover fashion, or fashionistas discover engineering. 31ectromode articulates itself as a kind of bridge between fashion and technology. 
Acknowledgments. I would like to thank Christopher L. Salter, Joanna Berzowska, Patrick H. Harrop, Anouk Wipprecht, and Isabelle Campeau for their participation in sharing ideas and expertise as well as acting as idea bouncing boards.

\section{References}

1. Entwistle, J.: The Fashioned Body: Fashion, Dress and Modern Social Theory. Polity, Cambridge (2000)

2. Lipovetsky, G.: The Empire of Fashion: Dressing Modern Democracy (Porter, C., trans.). Princeton University Press, Princeton \& Oxford (1994)

3. Troy, N.J.: Couture Culture: A Study in Modern Art \& Fashion. The MIT Press, Cambridge \& London (2003)

4. White, P.: Poiret. Studio Vista, London (1985)

5. Wilson, E.: Adorned in Dreams: Fashion and Modernity. Virago, London (1985)

6. Berman, M.: All That Is Solid Melts Into Air. Penguin, London (1988)

7. Kern, S.: The Culture of Time and Space: 1880-1919. Harvard University Press, Cambridge \& London (1991)

8. Lehmann, U.: Tigersprung: Fashion in Modernity. The MIT Press, Cambridge \& London (2000)

9. Breward, C., Evans, C. (eds.): Fashion and Modernity. Berg, Oxford \& New York (2005)

10. Sennet, R.: The Craftsman. Yale University Press, New Haven \& London (2008)

11. Andersen, C.: Makers: The New Industrial Revolution. McClelland \& Stewart, Toronto (2012)

12. Gershenfeld, N.: FAB: The Coming Revolution on Your Desktop-from Personal Computers to Personal Fabrication. Basic Books, New York (2005)

13. Igoe, T., O’Sullivan, D.: Physical Computing: Sensing and Controlling the Physical World with Computers. Thomson Course Technology, Boston (2004)

14. Able, B.V., Evers, L., Klaassen, R., Troxler, P. (eds.): Open Design Now. Waag Society, Amsterdam (2011)

15. Barnard, M.: Fashion as Communication. Routledge, London \& New York (2002) 